\title{
Clinical Pathways of Patients Denied Total Knee Arthroplasty Due to an Institutional BMI Cutoff
}

\author{
Charlie D. Wilson, MD ${ }^{1,2}$ Kathleen F. Lundquist, MD ${ }^{2}$ Nathan H. Baruch, BS ${ }^{2}$ \\ Ravindra Gaddipati, BS ${ }^{2}$ Kendall A.P. Hammonds, MPH $^{3}$ Bryce C. Allen, MD ${ }^{1,2}$
}

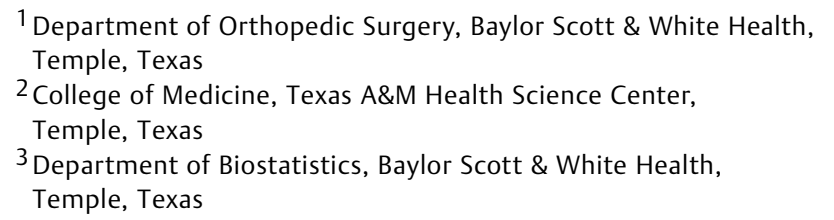

Address for correspondence Charlie D. Wilson, MD, Department of Orthopedic Surgery, Baylor Scott \& White Health, 5612 Drury Lane, Temple, TX 76502 (e-mail: cdwilson0721@gmail.com).

J Knee Surg 2022;35:1364-1369.

\begin{abstract}
Keywords

- BMI cutoff

- obesity

- total knee arthroplasty

- knee arthroplasty

- weight loss
\end{abstract}

As a result of the rising prevalence of obesity in North America, an increasing number of morbidly obese patients with primary knee osteoarthritis will be evaluated in need of total knee arthroplasty (TKA). ${ }^{1,2}$ However, many studies have established a link between obesity and complications from TKA. ${ }^{3-10}$ In 2013, the American Association of Hip and Knee Surgeons (AAHKS) Evidence-Based Committee

received

June 12, 2020

accepted after revision

January 2, 2021

published online

February 19, 2021

published the following statement: "The morbidly obese $\left(\mathrm{BMI}>40 \mathrm{~kg} / \mathrm{m}^{2}\right)$ and the super obese $\left(\mathrm{BMI}>50 \mathrm{~kg} / \mathrm{m}^{2}\right)$ have complication profiles that may outweigh the functional benefits of total joint arthroplasty (TJA). These patients should be counseled regarding these risks prior to any surgical intervention. It is our consensus opinion that consideration should be given to delaying TJA in a patient

(c) 2021. Thieme. All rights reserved. Thieme Medical Publishers, Inc., 333 Seventh Avenue, 18th Floor, New York, NY 10001, USA
DOI https://doi.org/ 10.1055/s-0041-1723969. ISSN 1538-8506. 
with a BMI $>40 \mathrm{~kg} / \mathrm{m}^{2}$, especially when associated with other comorbid conditions, such as poorly controlled diabetes or malnutrition."11 As a result, many institutions have established a BMI cutoff, over which patients are not eligible for TKA. ${ }^{12}$ However, the degree to which surgeons adhere to their institution's BMI cutoffs has not been welldefined in the literature, and it is possible that surgeons take significant leeway.

The ostensible utility of an institutional BMI cutoff is to mitigate the increased risk of complications associated with performing a TKA in the morbidly obese. Caring for morbidly obese patients is costly. ${ }^{13-15}$ With increasing emphasis on value-based care in the United States, these considerations are especially important. ${ }^{16}$ It is unclear whether an investment in clinic time and resources for evaluation of the morbidly obese that initially do not meet a BMI cutoff will lead to patients achieving weight loss goals to become surgical candidates. Few studies have examined the percentage of patients over a BMI cutoff that clinicians can expect to return for surgery once they have established care. ${ }^{17,18}$

In the current study, we aimed to identify the percentage of morbidly obese patients (in other words, those over the institutional BMI cutoff) who present with a diagnosis of primary knee osteoarthritis and ultimately undergo TKA at a BMI less than $40 \mathrm{~kg} / \mathrm{m}^{2}$ within 2 years of their initial visit. As secondary goals, we aimed to elucidate what percentage of patients underwent TKA at a BMI greater than $40 \mathrm{~kg} / \mathrm{m}^{2}$ or went to an outside hospital to have surgery, and in patients who did not undergoTKA, what percentage of patients reached a BMI less than $40 \mathrm{~kg} / \mathrm{m}^{2}$ within 2 years of their initial visit. This information could help surgeons make informed decisions about the deployment of clinic resources, aid in patient counseling, and establish a baseline of expected outcomes for future studies looking to examine the utility of a BMI cutoff and the fate the morbidly obese who are not offered a TKA.

\section{Materials and Methods}

We performed an observational study in which we queried the institutional electronic medical record (EMR) (Epic Systems Corporation, Verona, WI) to identify morbidly obese patients 40 years of age and older with a diagnosis of knee osteoarthritis who presented to a high-volume arthroplasty clinic for surgical evaluation. Morbid obesity was defined as BMI greater than $40 \mathrm{~kg} / \mathrm{m}^{2}$ calculated using the height and weight data gathered by the medical assistant at the patient's initial clinic visit. The query was performed for patient presenting between February 2014 (when the EMR was implemented) to March 2018 (to allow for 2 years of follow-up). We initially identified and screened 775 patients. Patients were excluded if they presented with a diagnosis other than primary knee osteoarthritis, were less than 40 years old, had BMI less than $40 \mathrm{~kg} / \mathrm{m}^{2}$ on initial presentation, or had been seen outside of established date range. Our clinic is a multi-subspecialty practice, including sports medicine surgeons and nonsurgeon providers. We included only those patients who established care with an arthroplasty surgeon or physician assistant ( 5 total surgeons and 1 physician assistant). In an attempt to remove patients who continuously return to arthroplasty providers for injections or medications without a desire for surgery, we also excluded patients whose first EMR-recorded visit was listed as a "return visit" rather than a new patient encounter. Ultimately, 487 patients were excluded and 288 patients were included (-Fig. 1).

For these 288 patients, the EMR was used to record multiple demographic variables including diagnosis of diabetes, current smoking status, and prior history of bariatric surgery. In an attempt to characterize the frequency with which particular weight loss interventions are recommended on an initial clinic visit, we recorded whether there was documentation of patient counseling (including patients simply being told they were above the BMI cutoff), physical therapy referral, dietitian referral, or bariatric surgery referral. Our institution does not have an established weight loss protocol for morbidly obese patients; it is up to each provider to make weight loss recommendations and order interventions.

Patients' records were then examined for whether the patient ultimately underwent TKA at our institution within 2 years of their initial clinic visit. The patient's BMI at the time of surgery was recorded. Patients that did not undergo surgery at our institution were contacted by telephone or their medical record was reviewed to ascertain whether they had undergone TKA at an outside hospital within 2 years of their initial clinic visit. Thirty-two of 288 patients (11.1\%) were unable to be contacted and were considered lost to follow-up (-Fig. 1).

At our institution, height and weight are recorded for each clinical encounter, regardless of specialty. Thus, for each encounter in the EMR after the initial clinic visit, BMI was assessed. If BMI was recorded as less than $40 \mathrm{~kg} / \mathrm{m}^{2}$ at any time point, the patient was classified as "TKA-eligible," even if the visit of record was not with an orthopedist or the weight loss was not sustained.

Sample characteristics were described using descriptive statistics. Frequencies and percentages were used to describe categorical variables. Means and standard deviations or medians and ranges were used to describe continuous variables.

\section{Results}

Patient demographics are shown in - Table 1. The frequency of documentation of specific interventions is shown in - Table 2. Documentation of referrals to a physical therapy, dietitian, or bariatric surgeon was found in less than $6 \%$ of patients. Providers documented counseling patients on weight loss strategies or weight loss goals to qualify for TKA $47.6 \%$ of the time.

Overall, 32.4\% of patients underwent TKA within 2 years (-Table 3). The average BMI at initial visit in surgical patients was $43.6 \mathrm{~kg} / \mathrm{m}^{2}$ (40-58.33) versus $46.8 \mathrm{~kg} / \mathrm{m}^{2}$ (40-64.6) in the nonsurgical patients. For the surgical group, the average BMI decreased to $42.3 \mathrm{~kg} / \mathrm{m}^{2}(38.3-52.7)$ on the day of surgery. - Table 4 demonstrates the breakdown of surgical and nonsurgical patients who were able to achieve the weight loss to meet the institutional goal of BMI less than 


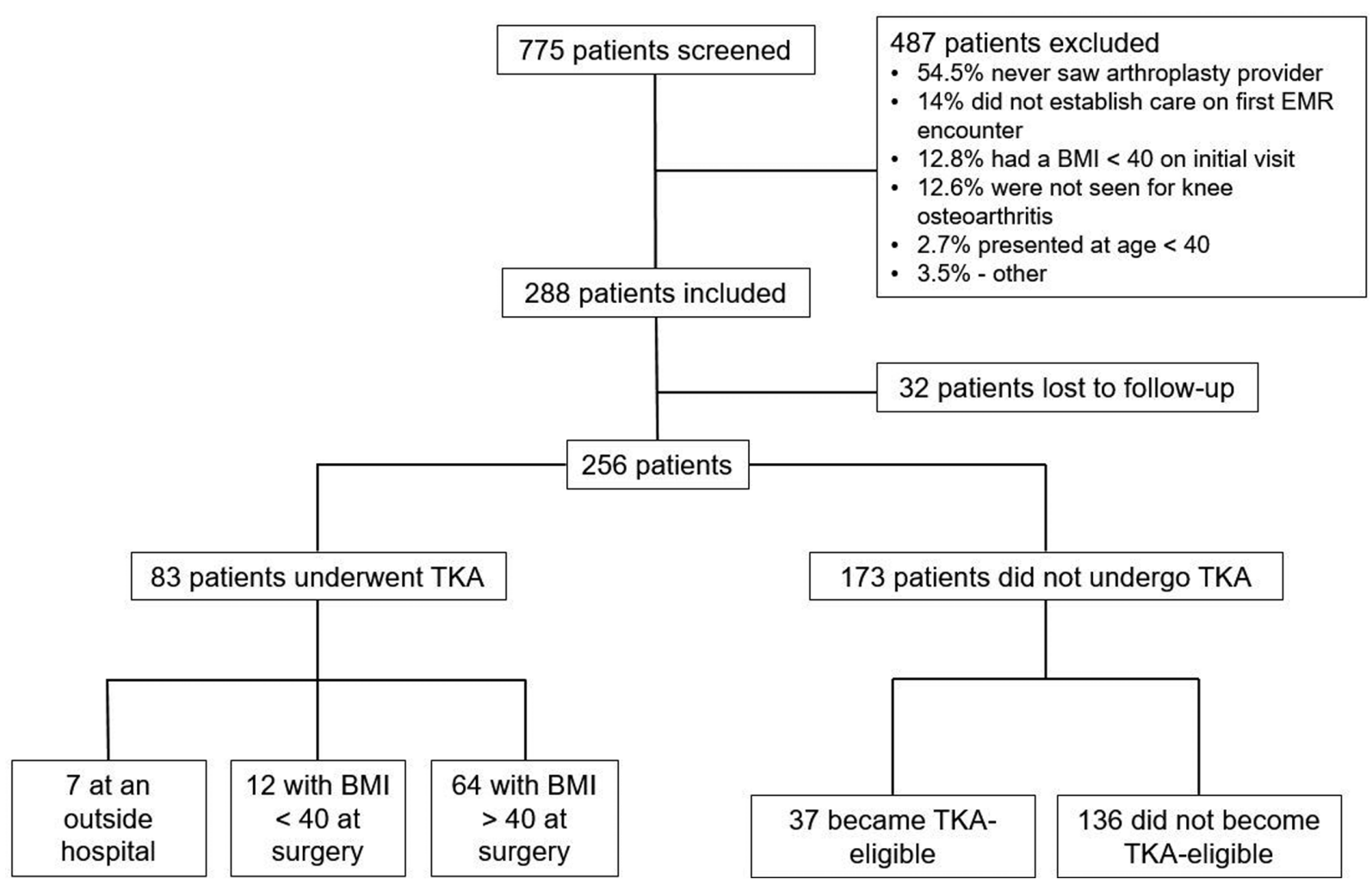

Fig. 1 Flow diagram showing the breakdown of patients excluded from and included in the study and the pathways of the included patients. $\mathrm{BMI}$, body mass index; EMR, electronic medical record; TKA, total knee arthroplasty.

Table 1 Patient demographics $(n=288)$

\begin{tabular}{|l|l|}
\hline Average age in years (range) & $59(40-84)$ \\
\hline Average BMI in kg/m² (range) & $46.2(40-65.4)$ \\
\hline Sex & Number (\% of total) \\
\hline Male & $83(28.8)$ \\
\hline Female & $205(71.2)$ \\
\hline Diagnosis of diabetes? & \\
\hline Yes & $125(43.4)$ \\
\hline No & $163(56.6)$ \\
\hline Current smoker? & \\
\hline Yes & $28(9.7)$ \\
\hline No & $260(90.3)$ \\
\hline Prior history of bariatric surgery? & \\
\hline Yes & $21(7.3)$ \\
\hline No & $267(92.7)$ \\
\hline
\end{tabular}

Abbreviation: BMI, body mass index.

$40 \mathrm{~kg} / \mathrm{m}^{2}$. Of the 256 patients included in the study, 49 of 256 patients (19.1\%) managed to dip under the BMI cutoff and 12 of 256 actually underwent TKA at a BMI less than $40 \mathrm{~kg} / \mathrm{m}^{2}$ (4.7\%). Sixty-four of 256 patients (25\%) underwent TKA at BMI greater than $40 \mathrm{~kg} / \mathrm{m}^{2}$. Thirty-seven of 256 patients (14.4\%) became TKA-eligible but did not undergo surgery, while 136 of 256 patients (53.1\%) neither met the institutional cutoff of BMI $40 \mathrm{~kg} / \mathrm{m}^{2}$ nor had surgery within 2 years (-Fig. 1). Seven patients underwent TKA at outside institu-
Table 2 Frequency of interventions utilized by clinicians $(n=288)$

\begin{tabular}{|l|l|}
\hline Referrals placed & Number (\% of total) \\
\hline Physical therapy & $6(2.1)$ \\
\hline Dietitian & $2(0.7)$ \\
\hline Bariatric surgery & $7(2.4)$ \\
\hline $\begin{array}{l}\text { Was there counseling/ } \\
\text { discussion of BMI cutoff? }\end{array}$ & $137(47.6)$ \\
\hline Yes & $151(52.4)$ \\
\hline No & \\
\hline
\end{tabular}

tions and BMI at the time of surgery could not ascertained. - Table 5 depicts our primary findings stratified according to BMI.

\section{Discussion}

The current study assessed the clinical pathways of morbidly obese patients with knee osteoarthritis who establish care with an arthroplasty practice with an institutional BMI cutoff of $40 \mathrm{~kg} / \mathrm{m}^{2}$, by determining the percentage of patients who undergo surgery and the percentage of patients that lose enough weight to be considered "eligible" according to an institutional BMI cutoff. We also attempted to define provider tendencies in the initial management of morbidly obese patients based on documented recommendations and interventions. 
Table 3 Surgical pathways of all patients $(n=256)$

\begin{tabular}{|l|l|l|l|}
\hline Underwent TKA & Number (\% of total) & $\begin{array}{l}\text { Average BMI in } \mathrm{kg} / \mathrm{m}^{2} \\
\text { at initial visit (range) }\end{array}$ & $\begin{array}{l}\text { Average BMI in } \mathrm{kg} / \mathrm{m}^{2} \text { on day } \\
\text { of surgery (range) }\end{array}$ \\
\hline No & $173(67.6)$ & $46.8(40-64.6)$ & - \\
\hline Yes & $83(32.4)$ & $43.6(40-58.33)$ & $42.3(38.3-52.7)$ \\
\hline
\end{tabular}

Abbreviations: BMI, body mass index; TKA, total knee arthroplasty.

Table 4 Clinical pathways of all patients relative to BMI cutoff $(n=256)$

\begin{tabular}{|l|l|}
\hline TKA performed within 2 years & $\begin{array}{l}\text { Number } \\
\text { (\% of total) }\end{array}$ \\
\hline BMI less than $40 \mathrm{~kg} / \mathrm{m}^{2}$ & $12(4.7)$ \\
\hline BMI greater than $40 \mathrm{~kg} / \mathrm{m}^{2}$ & $64(25)$ \\
\hline Surgery done at an outside hospital & $7(2.7)$ \\
\hline TKA not performed within 2 years & $37(14.5)$ \\
\hline $\begin{array}{l}\text { Became TKA-eligible } \\
\text { (BMI less than } 40 \mathrm{~kg} / \mathrm{m}^{2} \text { at any time) }\end{array}$ & $136(53.1)$ \\
\hline $\begin{array}{l}\text { Did not become TKA-eligible } \\
\text { (BMI never less than } 40 \mathrm{~kg} / \mathrm{m}^{2} \text { ) }\end{array}$ \\
\hline
\end{tabular}

Abbreviations: BMI, body mass index; TKA, total knee arthroplasty.

Table 5 Pathways of patients based on BMI strata

\begin{tabular}{|c|l|l|l|l|}
\hline & \multicolumn{3}{|l|}{ BMI on initial } \\
presentation $\left(\mathrm{kg} / \mathrm{m}^{2}\right)$ & \\
\hline & $40-45$ & $45-50$ & $50+$ & Total \\
\hline $\begin{array}{l}\text { Number of patients who } \\
\text { did not undergo TKA }\end{array}$ & 79 & 58 & 36 & 173 \\
\hline $\begin{array}{c}\text { Number who became } \\
\text { TKA-eligible }\end{array}$ & 31 & 5 & 1 & 37 \\
\hline $\begin{array}{c}\text { Number who did not } \\
\text { become TKA-eligible }\end{array}$ & 48 & 53 & 35 & 136 \\
\hline $\begin{array}{l}\text { Number of patients who } \\
\text { underwent TKA }\end{array}$ & 61 & 19 & 3 & 83 \\
\hline $\begin{array}{l}\text { Average BMI at } \\
\left.\text { surgery (kg/m }{ }^{2}\right)\end{array}$ & 41.4 & 44.7 & 46.6 & - \\
\hline
\end{tabular}

Abbreviations: BMI, body mass index; TKA, total knee arthroplasty.

A few previous studies have examined similar patient populations. Springer et al prospectively enrolled 289 morbidly obese patients who presented to clinic with both hip and knee osteoarthritis. Their study differed in that they provided an intervention for all patients: a referral to a bariatric surgeon. Only $23.2 \%$ of patients saw the bariatric surgeon, and of those, just $20.9 \%$ had bariatric surgery. Ultimately, $20.1 \%$ of their original cohort underwent TJA at an average BMI of $42.3 \mathrm{~kg} / \mathrm{m}^{2}$. Of the patients who did have a TJA, 39.7\% had a BMI less than 40 at the time of surgery. The authors did not attempt to ascertain whether patients may have had surgery at an outside hospital. ${ }^{18}$ Shapiro et al retrospectively examined 99 morbidly obese patients who were denied TKA. Of those, $20.2 \%$ met the target weight and underwent TKA at their institution. Eight of 99 patients (8.1\%) underwent TKA at another institution. ${ }^{17}$ The current study reports findings similar to these historical precedents. However, we also report the weight loss success of all patients who were reviewed, not just those who underwent TKA. Of the 173 patients who did not undergo TKA, 37 patients (21.4\%) successfully brought their BMI to less than $40 \mathrm{~kg} / \mathrm{m}^{2}$.

The current study is limited by its unclear generalizability to other practices. Regional demographics and barriers to access affect the patient population that any given provider sees in clinic. Our institution is a large academic medical center in the midst of a relatively poor, rural area ${ }^{19}$ in a state with one of the highest prevalence of obese patients. ${ }^{20}$ Multiple nonsurgeon providers often serve as an initial screen to offload the clinical burden of our surgeons. As a result, the patients that surgeons see in clinic typically have expressed at least some level of inclination toward surgical intervention. Compared with a practice without such screening measures, our clinical setup may therefore overestimate the number of morbidly obese patients an arthroplasty surgeon may expect to undergo surgery within 2 years. After all, 487 patients were excluded from analysis, the majority for not having seen an arthroplasty surgeon or physician assistant.

The current study is also limited by its retrospective nature. While scheduling an appointment with an arthroplasty surgeon when multiple nonoperative provider options exist demonstrates a certain level of initiative, we cannot ensure that all patients would have met surgical criteria for arthroplasty if not for their BMI, or if each patient would have elected to proceed with surgery had it been offered at the initial visit. However, the surgical discussion is often nuanced, and even a prospective study would be subject to bias as operative criteria would inevitably vary subtly from surgeon to surgeon, and there would be no way to identify which patients truly desire surgery if they were precluded by their BMI.

The retrospective nature of this study also makes it difficult to draw conclusions based on the percentages of patients who were provided with counseling or referrals because of the variability in documentation and the use of EMR templates among providers. Documentation of interventions or counseling may not accurately reflect the discussions held with the patient. Even so, discussion of BMI cutoffs and weight loss counseling would ideally approach $100 \%$. Institutional restrictions necessitate institutional solutions. As recent AAHKS symposium stated, "collaborative care pathways need to be developed to provide a comprehensive optimization program for patients who need total joint replacement."21 Our 
institution would likely benefit from an established weight-loss protocol from morbidly obese patients who desire TKA.

While there is an abundance of literature on the general effectiveness of different weight loss strategies, there is a relative dearth of studies examining interventions to prepare patients for TKA. Dieting protocols and dietitian supervision have shown promise. ${ }^{22,23}$ Aquatic exercise may be an effective form of physical activity. ${ }^{24}$ In addition to oral pain medications or steroid injections, genicular nerve ablation has been proposed as an alternative pain control approach, ${ }^{25}$ which could theoretically allow patients to exercise more effectively. Many patients with a BMI over $40 \mathrm{~kg} / \mathrm{m}^{2}$ are referred to bariatric surgery, ${ }^{18,26}$ which has been shown to be effective in helping patients achieve weight loss goals and may even obviate the need for TKA in some patients. ${ }^{27}$ Our institution has not developed any systematic weight loss protocols for patients, and therefore it is up to individual providers to counsel patients on effective weight loss strategies and provide referrals for specific weight loss interventions. Adherence to robust, evidence-based protocols has been effective in addressing other perioperative challenges, ${ }^{28}$ so development and implementation of weight loss protocols prior to elective TKA may benefit morbidly obese patients.

There is very little evidence in the literature about provider adherence to institutional BMI cutoffs. Shapiro et al offered surgery to patients who make a "concerted attempt" at weight loss (defined as at least reaching two-thirds of the weight loss goal). ${ }^{17}$ Our institution varies by provider, but in general, most of our surgeons do make concessions for those patients who demonstrate motivation to lose weight, such as achieving a significant portion of a weight loss goal or losing $10 \%$ of total body weight, even if those patients ultimately do not reach the BMI cutoff of $40 \mathrm{~kg} / \mathrm{m}^{2}$. These habits help explain why the average BMI of the surgical patients was $42.4 \mathrm{~kg} / \mathrm{m}^{2}$ in this study. Future research to define the strictness with which surgeons adhere to their institution's BMI cutoffs would likely be of significant interest to the arthroplasty community.

Interestingly, fewer patients in the current study went "doctor-shopping" than the authors anticipated. Doctor-shopping involves visiting multiple doctors for the treatment of the same health condition. ${ }^{29}$ Morbidly obese patients have been shown to be up to $52 \%$ more likely to seek multiple primary care providers during a 2-year period. ${ }^{30}$ One might think that by withholding TKA, patients will simply find another surgeon nearby to do the surgery. Of the 180 patients who were not offered surgery at our institution, only 7 (3.9\%) patients underwent surgery elsewhere. Shapiro et al found that similarly, 8.1\% of patients underwent TKA at an outside institution. The prevalence of "surgeon-shopping" will depend in part upon the location of a practice and patients' ease of access to other surgeons. Even so, surgeon-shopping may not be as pervasive as one would expect, and the phenomenon merits further study.

The number of patients in the current study who were able to successfully lose weight, either to undergo TKA safely or to continue to live with their knee osteoarthritis was $19.1 \%$. As we understand the limited efficacy of self-directed weight loss attempts in the TJA population, delineation of evidence-based pathways to provide safe and effective care for these patients become critical. Myriad studies have shown an increase in complications, particularly infection, related to increasing BMI and TJA. ${ }^{3-10}$ However, it is also clear that morbidly obese patients benefit from TKA. ${ }^{31,32}$ Weight loss prior to TJA has been shown to decrease length of stay. ${ }^{33}$ While limited evidence suggests that weight loss would lower the risk of obesity-related complications, ${ }^{34,35}$ nonsurgical weight loss or undergoing bariatric surgery prior to TKA may have no effect on risks ${ }^{36,37}$ or actually increase such risks. ${ }^{38-40}$ Studies currently underway may shed some more light on this conundrum. ${ }^{41}$ Future studies will address the costs and benefits of an institutional BMI cutoff, the utility of particular weight loss interventions, and the impact of weight loss prior to TKA.

\section{Conclusions}

With the increasing incidence of obesity in the United States, it is important to consider the potential clinical courses of morbidly obese patients with knee osteoarthritis. This descriptive study shows that without strict enforcement of a BMI cutoff and a lack of specific weight loss strategies for patients, the number is patients who reach the sub- $40 \mathrm{~kg} / \mathrm{m}^{2}$ BMI milestone is low. This information may be used to further evaluate the utility of an institutional BMI cutoff and investigate strategies to more effectively usher patients to a lower BMI prior to surgery.

\section{Ethical Approval}

Ethical approval was provided by Baylor Scott \& White Research IRB (Project ID 018-534, Reference Number: 315799).

Conflict of Interest

None declared.

\section{References}

1 Bourne R, Mukhi S, Zhu N, Keresteci M, Marin M. Role of obesity on the risk for total hip or knee arthroplasty. Clin Orthop Relat Res 2007;465(465):185-188

2 Wendelboe AM, Hegmann KT, Biggs JJ, et al. Relationships between body mass indices and surgical replacements of knee and hip joints. Am J Prev Med 2003;25(04):290-295

3 Adhikary SD, Liu WM, Memtsoudis SG, Davis CM III, Liu J. Body mass index more than $45 \mathrm{~kg} / \mathrm{m}(2)$ as a cutoff point is associated with dramatically increased postoperative complications in total knee arthroplasty and total hip arthroplasty. J Arthroplasty 2016; 31(04):749-753

4 Boyce L, Prasad A, Barrett M, et al. The outcomes of total knee arthroplasty in morbidly obese patients: a systematic review of the literature. Arch Orthop Trauma Surg 2019;139(04): 553-560

5 Dowsey MM, Liew D, Stoney JD, Choong PF. The impact of preoperative obesity on weight change and outcome in total knee replacement: a prospective study of 529 consecutive patients. J Bone Joint Surg Br 2010;92(04):513-520

6 Everhart JS, Altneu E, Calhoun JH. Medical comorbidities are independent preoperative risk factors for surgical infection after total joint arthroplasty. Clin Orthop Relat Res 2013;471(10):3112-3119 
7 Prohaska MG, Keeney BJ, Beg HA, et al. Preoperative body mass index and physical function are associated with length of stay and facility discharge after total knee arthroplasty. Knee 2017;24(03):634-640

8 Si HB, Zeng Y, Shen B, et al. The influence of body mass index on the outcomes of primary total knee arthroplasty. Knee Surg Sports Traumatol Arthrosc 2015;23(06):1824-1832

9 Sisko ZW, Vasarhelyi EM, Somerville LE, Naudie DD, MacDonald SJ, McCalden RW. Morbid obesity in revision total knee arthroplasty: a significant risk factor for re-operation. J Arthroplasty 2019;34(05):932-938

10 Ward DT, Metz LN, Horst PK, Kim HT, Kuo AC. Complications of morbid obesity in total joint arthroplasty: risk stratification based on BMI. J Arthroplasty 2015;30(9, Suppl):42-46

11 Workgroup of the American Association of Hip and Knee Surgeons Evidence Based Committee. Obesity and total joint arthroplasty: a literature based review. J Arthroplasty 2013;28(05):714-721

12 McElroy MJ, Pivec R, Issa K, Harwin SF, Mont MA. The effects of obesity and morbid obesity on outcomes in TKA.J Knee Surg 2013; 26(02):83-88

13 Dowsey MM, Liew D, Choong PF. Economic burden of obesity in primary total knee arthroplasty. Arthritis Care Res (Hoboken) 2011;63(10):1375-1381

14 Girardi FM, Liu J, Guo Z, Valle AGD, MacLean C, Memtsoudis SG. The impact of obesity on resource utilization among patients undergoing total joint arthroplasty. Int Orthop 2019;43(02):269-274

15 Kremers HM, Visscher SL, Kremers WK, Naessens JM, Lewallen DG. The effect of obesity on direct medical costs in total knee arthroplasty. J Bone Joint Surg Am 2014;96(09):718-724

16 Urish KL, Qin Y, Li BY, et al. Predictors and cost of readmission in total knee arthroplasty. J Arthroplasty 2018;33(09):2759-2763

17 Shapiro JA, Narayanan AS, Taylor PR, Olcott CW, Del Gaizo DJ. Fate of the morbidly obese patient who is denied total joint arthroplasty. J Arthroplasty 2020;35(6S):S124-S128

18 Springer BD, Roberts KM, Bossi KL, Odum SM, Voellinger DC. What are the implications of withholding total joint arthroplasty in the morbidly obese? A prospective, observational study. Bone Joint J 2019;101-B(7_Supple_C):28-32

19 Texas Comptroller of Public Accounts "The Central Texas Region 2018 Regional Report.” The Central Texas Region, 2018, comptroller.texas.gov/economy/economic-data/regions/central.php

20 Le A, Judd SE, Allison DB, et al. The geographic distribution of obesity in the US and the potential regional differences in misreporting of obesity. Obesity (Silver Spring) 2014;22(01):300-306

21 Springer BD. Management of the bariatric patient. what are the implications of obesity and total joint arthroplasty: the orthopedic surgeon's perspective? J Arthroplasty 2019;34(7S):S30-S32

22 Gandler N, Simmance N, Keenan J, Choong PF, Dowsey MM. A pilot study investigating dietetic weight loss interventions and 12 month functional outcomes of patients undergoing total joint replacement. Obes Res Clin Pract 2016;10(02):220-223

23 Liljensøe A, Laursen JO, Bliddal H, Søballe K, Mechlenburg I. Weight loss intervention before total knee replacement: a 12-month randomized controlled trial. Scand J Surg 2019;14574969198838 12:1457496919883812. Doi: 10.1177/1457496919883812

24 Yázigi F, Espanha M, Vieira F, Messier SP, Monteiro C, Veloso AP. The PICO project: aquatic exercise for knee osteoarthritis in overweight and obese individuals. BMC Musculoskelet Disord 2013;14:320

25 Iannaccone F, Dixon S, Kaufman A. A review of long-term pain relief after genicular nerve radiofrequency ablation in chronic knee osteoarthritis. Pain Physician 2017;20(03):E437-E444
26 McLawhorn AS, Southren D, Wang YC, Marx RG, Dodwell ER. Costeffectiveness of bariatric surgery prior to total knee arthroplasty in the morbidly obese: a computer model-based evaluation. J Bone Joint Surg Am 2016;98(02):e6. Doi: 10.2106/JBJS.N.00416

27 Rishi L, Bhandari M, Kumar R. Can bariatric surgery delay the need for knee replacement in morbidly obese osteoarthritis patients. J Minim Access Surg 2018;14(01):13-17

28 Auyong DB, Allen CJ, Pahang JA, Clabeaux JJ, MacDonald KM, Hanson NA. Reduced length of hospitalization in primary total knee arthroplasty patients using an updated enhanced recovery after orthopedic surgery (ERAS) pathway. J Arthroplasty 2015;30 (10):1705-1709

29 Biernikiewicz M, Taieb V, Toumi M. Characteristics of doctor-shoppers: a systematic literature review. J Mark Access Health Policy 2019;7(01):1595953. Doi: 10.1080/20016689.2019.1595953

30 Gudzune KA, Bleich SN, Richards TM, Weiner JP, Hodges K, Clark JM. Doctor shopping by overweight and obese patients is associated with increased healthcare utilization. Obesity (Silver Spring) 2013;21(07):1328-1334

31 Baker P, Petheram T, Jameson S, Reed M, Gregg P, Deehan D. The association between body mass index and the outcomes of total knee arthroplasty. J Bone Joint Surg Am 2012;94(16):1501-1508

32 Hakim J, Volpin G, Amashah M, et al. Long-term outcome of total knee arthroplasty in patients with morbid obesity. Int Orthop 2020;44(01):95-104

33 Keeney BJ, Austin DC, Jevsevar DS. Preoperative weight loss for morbidly obese patients undergoing total knee arthroplasty: determining the necessary amount. J Bone Joint Surg Am 2019; 101(16):1440-1450

34 Li S, Luo X, Sun H, Wang K, Zhang K, Sun X. Does prior bariatric surgery improve outcomes following total joint arthroplasty in the morbidly obese? A meta-analysis. J Arthroplasty 2019;34(03): 577-585

35 Werner BC, Kurkis GM, Gwathmey FW, Browne JA. Bariatric surgery prior to total knee arthroplasty is associated with fewer postoperative complications. J Arthroplasty 2015;30(9, Suppl):81-85

36 Inacio MC, Kritz-Silverstein D, Raman R, et al. The impact of preoperative weight loss on incidence of surgical site infection and readmission rates after total joint arthroplasty. J Arthroplasty 2014;29(03):458-64.e1

37 Smith TO, Aboelmagd T, Hing CB, MacGregor A. Does bariatric surgery prior to total hip or knee arthroplasty reduce postoperative complications and improve clinical outcomes for obese patients? Systematic review and meta-analysis. Bone Joint J 2016; 98-B(09):1160-1166

38 Lee GC, Ong K, Baykal D, Lau E, Malkani AL. Does prior bariatric surgery affect implant survivorship and complications following primary total hip arthroplasty/total knee arthroplasty? J Arthroplasty 2018;33(07):2070-2074.e1

39 Lui M, Jones CA, Westby MD. Effect of non-surgical, non-pharmacological weight loss interventions in patients who are obese prior to hip and knee arthroplasty surgery: a rapid review. Syst Rev 2015;4:121. Doi: 10.1186/s13643-015-0107-2

40 Meller MM, Goodman S, Gonzalez MH, Lau E. Does bariatric surgery normalize risks after total knee arthroplasty? Administrative Medicare data. J Am Acad Orthop Surg Glob Res Rev 2019;3 (12):e19.00102. Doi: 10.5435/JAAOSGlobal-D-19-00102

41 Benotti PN, Still CD, Craig Wood G, et al;SWIFT Trial Investigators. Surgical weight-loss to improve functional status trajectories following total knee arthroplasty: SWIFT trial: rationale, design, and methods. Contemp Clin Trials 2018;69:1-9 